\title{
In-Depth Analysis of Supramolecular Interfacial Polymerization via a Computer Simulation Strategy
}

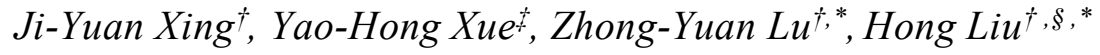

$\uparrow$ State Key Laboratory of Supramolecular Structure and Materials, Institute of Theoretical Chemistry, Jilin University, Changchun, 130023, China;

$\ddagger$ Information Science School, Guangdong University of Finance and Economics, Guangzhou 510320, China;

$\S$ Key Laboratory of Theoretical Chemistry of Environment Ministry of Education, School of Chemistry and Environment, South China Normal University, Guangzhou 510006, China E-mail: 1uzhy@,jlu.edu.cn; hongliu@m.scnu.edu.cn 

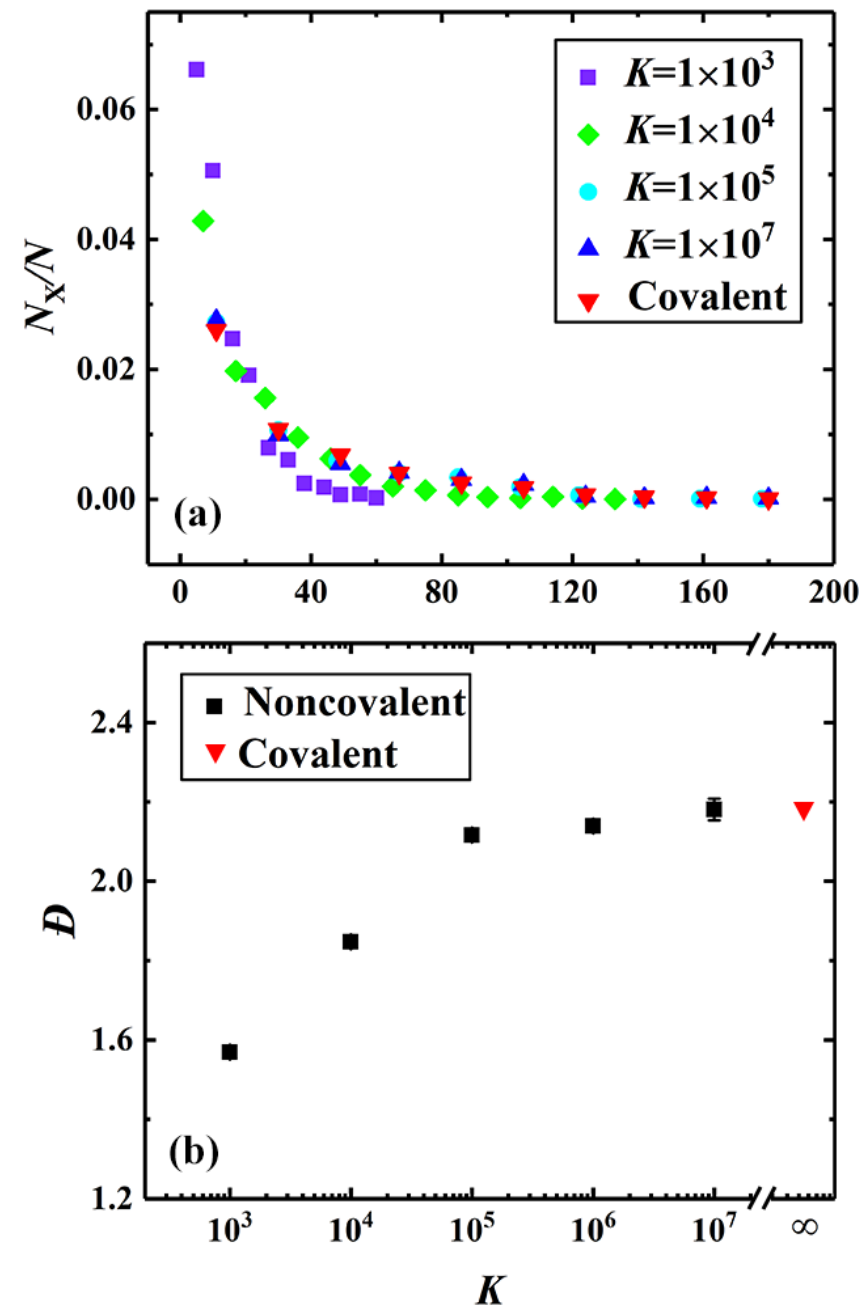

Fig. S1 (a) The number fraction distributions with different association ratio values $K$. (b) The dependence of the polydispersity index of the final products on the association ratio value $K$. 


\section{The relation between the association of the supramolecular bond and the result}

Regarding to the relation between the association constant of the supramolecular bond and the results, we make the following simulation validations. We define a fully breaking probability of the quadruple hydrogen bond as $P_{\mathrm{r}^{-}}$, so that a simple association ratio, i.e., $K$ $=P_{\mathrm{r}} / P_{\mathrm{r}^{-}}$, can be defined. Here $P_{\mathrm{r}}$ is the preset reaction probability of bonding, which is defined as $P_{\mathrm{r}}=0.01$ in the present study. On concerning that the bond formation and breaking share the same reaction interval as stated in the manuscript, the association ratio $K$ should partially represent the association constant value. In the following Fig. 1a, we show the number fraction distributions with different association ratio values $K$. In this figure, the "covalent" means that $P_{\mathrm{r}^{-}}=0$, i.e., the quadruple hydrogen bond is not possible to fully break up. It is clearly shown in Fig. 1a that, with $K=1 \times 10^{3} \sim 1 \times 10^{5}$, the number fraction distributions seem quite different. For $K=1 \times 10^{3}$, the number fraction of short chains (with low $X$ ) has obviously high value. That is because, relatively smaller $K$ corresponds to larger $P_{\mathrm{r}}^{-}$, which further leads to the high possibility of breaking of quadruple hydrogen bond. As a consequence, it is reasonable to find that the fraction of short chains (formed by breaking of polymerized long chains at the places of quadruple hydrogen bonds) is increased. Besides, we also find that when $K \geq 1 \times 10^{5}$, the number fraction distribution seems independent on $K$. On the other hand, Nakashima et al. ${ }^{1-2}$ and Meijer et al. ${ }^{3}$ had respectively pointed out that the binding constant of quadruple hydrogen bond is as high as the order of $10^{7}$, implying that the probability of its breaking is quite low. Based on the above analysis, for making our model easy to handle, we consider the quadruple hydrogen bond as typically impossible to break, i.e., $P_{\mathrm{r}^{-}}=0$.

A further validation is the dependence of the polydispersity index on the association ratio value $K$, as shown in Fig. 1b. It is apparent that the increasing trend of polydispersity index with $K$ reaches a plateau when $K \geq 1 \times 10^{5}$. This result again supports that our handling of irreversibility of quadruple hydrogen bond is reasonable to reproduce the true polymerization. 


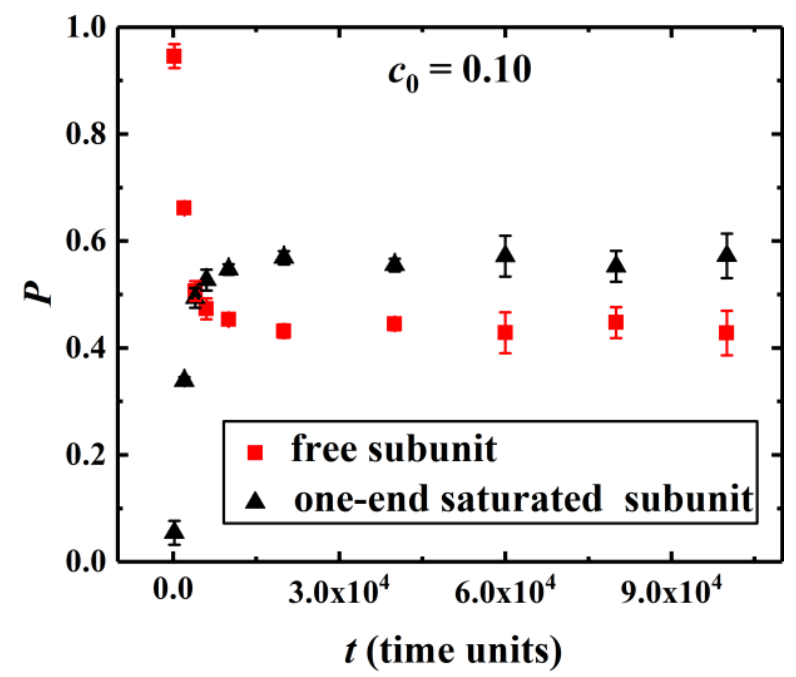

Fig. S2. Time evolution of the fractions of newly generated A-B bonds from free subunit monomers and from one-end saturated subunit monomers. 

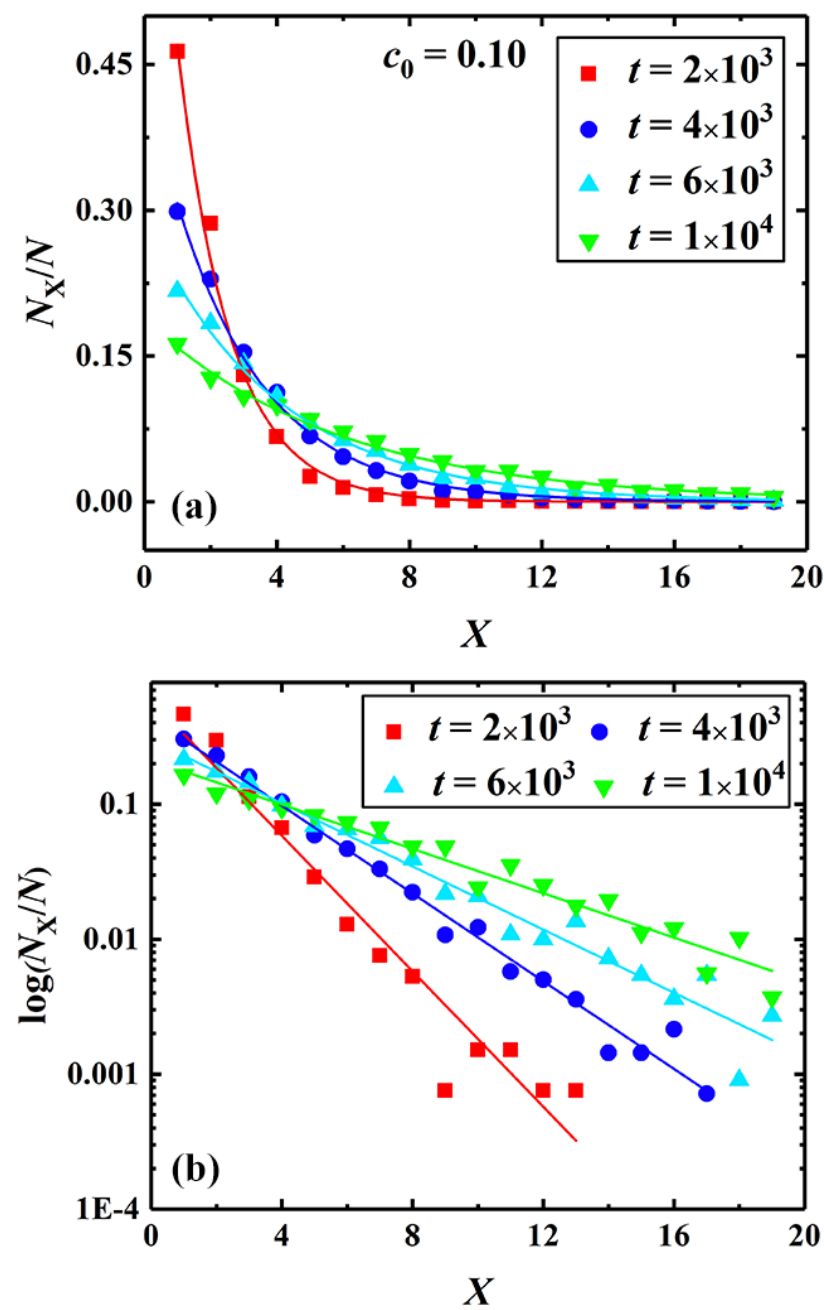

Fig. S3. (a) The number fraction distributions at different stages of supramolecular interfacial polymerization with $\mathrm{t}<2 \times 10^{4}$. (b) The linear relationship of $\log (N \mathrm{x} / N) \sim X$ that is reprocessed with the data of (a). 


\section{REFERENCES}

1. Park, T.; Todd, E. M.; Nakashima, S.; Zimmerman, S. C., A Quadruply Hydrogen Bonded Heterocomplex Displaying High-Fidelity Recognition. Journal of the American Chemical Society 2005, 127 (51), 18133-18142.

2. Park, T.; Zimmerman, S. C.; Nakashima, S., A Highly Stable Quadruply Hydrogen-Bonded Heterocomplex Useful for Supramolecular Polymer Blends. Journal of the American Chemical Society 2005, 127 (18), 6520-6521.

3. Sijbesma, R. P.; Beijer, F. H.; Brunsveld, L.; Folmer, B. J. B.; Hirschberg, J. H. K. K.; Lange, R. F. M.; Lowe, J. K. L.; Meijer, E. W., Reversible Polymers Formed from Self-Complementary Monomers Using Quadruple Hydrogen Bonding. Science 1997, 278 (5343), 1601-1604. 